\title{
ON THE APPROXIMATION OF INVARIANT SETS FOR CONSTRAINED LDT SYSTEMS
}

\author{
Paolo Caravani ${ }^{1}$
}

\author{
Electrical Engineering Department, University of L'Aquila, \\ Monteluco di Roio, 67040 L'Aquila,Italy \\ caravani@ing. univaq. it
}

\begin{abstract}
An algorithm is given for the computation of the maximal invariant subset of a given set for linear discrete-time systems subject to hard constraints on state, control and disturbance inputs. At each iteration the algorithm provides an inner invariant approximation of the maximal invariant set. Copyright ${ }^{\circledR} 2005$ IFAC.
\end{abstract}

Keywords: Constrained Systems, Control Invariance, Polyhedral Invariance

\section{INTRODUCTION}

Set invariance plays a fundamental role in the design of controllers for constrained systems (Blanchini [1999a]). Indeed, constraints on state and control are satisfied at all times if and only if the initial state is contained inside an invariant set.

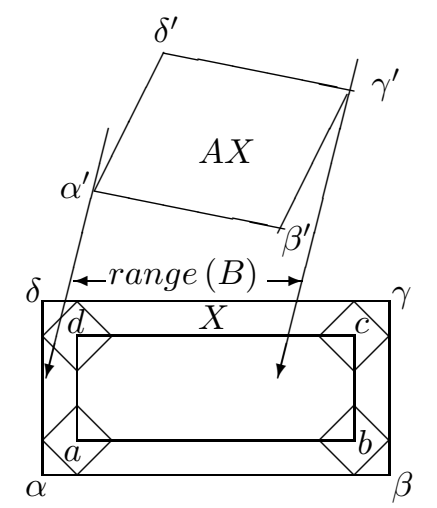

Fig. 1 Geometry of Invariant Set.

\footnotetext{
1 Support by MIUR under PRIN02 is gratefully acknowledged.
}

A control invariant ${ }^{2}$ set in discrete-time systems is a subset of state space such that for any state in it there exists a feasible control that keeps the successor state in it, for any admissible disturbance (feasible, admissible meaning control and disturbance satisfy constraints). A picture helps to illustrate the geometry of the invariance problem and serves as introduction to our approach. For $x^{+}=A x+B u+C v \in \mathbb{R}^{2}$ where $x^{+}$is the successor state assume $u \in U$ and $v \in V$ with $U, V$ given boxes. Box $X=\alpha \beta \gamma \delta$ (Fig. 1) is mapped by $A$ into $\alpha^{\prime} \beta^{\prime} \gamma^{\prime} \delta^{\prime}=A X$. In order for $X$ to be invariant, for each $z \in A X$ a vector $B u$ (with feasible $u$ ) must be found that maps $z$ inside $a b c d$, a subset of $X$ such that from any of its points the addition of a vector belonging to $C V$ does not lead outside $X$ (diamonds represent disturbance sets $C V$ added to the corners of $a b c d$ ). In Fig. 1 $X$ is not control invariant, as the range of $B$ is such that there is no $u$ mapping (for instance) point $\alpha^{\prime}$ inside $a b c d$. Notice, this is true regardless of the constraint on $u$. It is of interest in this case to determine whether $X$ contains a control invariant set, for state control and disturbance constraints

\footnotetext{
2 As we only deal with systems subject to control and disturbance constraints, we depart for brevity from the often used terminology robust or robustly controlled invariant.
} 
could be satisfied at all times starting from just such a set. The largest control invariant set would, loosely speaking, maximize chances of satisfying constraints. It is well known that a control invariant set contained in a closed bounded convex set $\mathcal{P}$ exists if and only if a maximal control invariant set $\hat{S}$ contained in $\mathcal{P}$ exists. The search for $\hat{S}$ can be done recursively by the repeated evaluation of the reach set $\mathcal{R}(X)$, the set of all states that reach $X$ in one step with a feasible control, for all admissible disturbances. The computation of $\mathcal{R}(X)$ entails set difference, projection, and intersection of convex sets. As the order of the system grows, these can be computationally expensive operations (see Veres [2002] for numerical aspects in polyhedral sets) whose simplification has attracted in the past considerable research effort:Lasserre [1993], Shamma [1996], Blanchini [1999b], De Santis [1997], Kerrigan [2000], Mayne [2001].

Two algorithms are available for the computation of $\hat{S}$. The first algorithm, known as external, builds up a family of sets $X_{k+1}=\mathcal{R}\left(X_{k}\right) \cap \mathcal{P}$ with $X_{0}=\mathcal{P}$. The second algorithm, known as internal, builds up a family of sets $X_{k+1}=\mathcal{R}\left(X_{k}\right) \cap$ $\mathcal{P}$ with $X_{0}=S$, where $S$ is a control invariant set contained in $\mathcal{P}$. Under broad conditions, both algorithms are known to converge to $\hat{S}$, the largest invariant subset of $\mathcal{P}$. The external algorithm generates sets with the property $X_{k+1} \subset X_{k}$, hence it approximates $\hat{S}$ from the outside. The internal algorithm generates sets with the property $X_{k+1} \supset X_{k}$, hence it approximates $\hat{S}$ from the inside (whence their name). The first algorithm has the drawback that the family of approximants is not invariant, so the successive approximations bear essentially worthless ${ }^{3}$ information; the second algorithm is preferable as each approximant is itself control invariant, thereby providing an approximate solution. The drawback however is that an initial control invariant set $S$ - with respect to a given $\mathcal{P}$ and given $V$ - must be found. This is easy to do in absence of disturbances, for in that case the equilibrium state $x_{e}$ is evidently invariant under $u_{e}$ such that $x_{e}=A x_{e}+B u_{e}$, and it can be assumed Gutman, Cwikel [1987], De Santis et al. [2004] $S=\left\{x_{e}\right\}$ as a starting point for the internal algorithm. However, we know of no general procedure to determine $S$ in presence of disturbances. This will indeed be one of the contributions of this paper.

Both algorithms suffer from the fact that convergence is not guaranteed to occur finitely, even if $\hat{S}$ is finitely generated. Moreover, even if $\mathcal{P}$ is a polyhedron $\widehat{S}$ need not be a polyhedron, e.g. a finitely generated set. The approach we take in this paper is based on the observation that if the

\footnotetext{
3 precisely, $X_{k}$ guarantees permanence in it for $k$ steps
}

range of the $B$ matrix is the entire space, then invariance of any set $\mathcal{P}$ depends purely on constraints and no longer on system structure: either the control constraints can make $\mathcal{P}$ invariant or, $\mathcal{P}$ cannot be made invariant. For polytopic $\mathcal{P}$ the test requires solving a system of linear inequalities - no iterations are necessary.

The price we pay for such a simplification is the need to consider $n$-step dynamics for a system of order $n$, so that the extended $B$ matrix (under reachability) has range $R^{n}$. Enforcing $n$-step invariance is a weaker condition than 1-step invariance (ordinary invariance) as temporary violations are allowed. Such a weakness ought to be compared with the approximations generated by the external algorithm which, when arrested at iteration $n$, only guarantee permanence in $X$ of trajectories of length $n$ at most. Here, we take the complementary view of allowing departure from $X$ of trajectories of length $n$ at most. Notice that our condition implies stable trajectories while the former does not. Moreover, weakness can be tempered by imposing that the maximal deviation from $X$ in steps $1 \ldots n-1$ be minimized. Should the deviation be zero, $X$ is invariant, hence we have a check of invariance not requiring set difference, projection, and intersection. Should $X$ be not invariant, the maximal deviation from $X$ defines a second set $\hat{X}$ such that trajectories starting in $X$ stay in $\hat{X}$. It turns out that this allows initialization of the internal algorithm, should the maximal invariant subset of $X$ be of interest. $\mathrm{No}$ tation: set inclusion $\mathcal{A} \subset \mathcal{B}$ is non-proper unless $\mathcal{A} \neq \mathcal{B}$ is specified. The interior of $\mathcal{A}$ is $\operatorname{int}(\mathcal{A})$, its convex hull $\operatorname{conv}(\mathcal{A})$. The set of vectors $x+y$ with $y \in \mathcal{B}$ is denoted $x+\mathcal{B}$, if in addition $x \in \mathcal{A}$, $\mathcal{A}+\mathcal{B}$ (Minkowski sum).

\section{PRELIMINARIES}

Consider systems described by

$$
x(t+1)=A x(t)+B u(t)+C v(t)
$$

where $t$ is an integer time-index, $x \in \mathbb{R}^{n}$ is the state, $u \in \mathbb{R}^{m}$ the control, $v \in \mathbb{R}^{p}$ the disturbance and matrices $A, B, C$ are assumed known. As we consider time invariant systems we often omit $t$ and use notation $x^{+}=A x+B u+C v$ where $x^{+}$is the successor state.

We define three sets, $X \subset \mathbb{R}^{n}, U \subset \mathbb{R}^{m}, V \subset \mathbb{R}^{p}$. A control is feasible if it takes values in $U$. A disturbance is admissible if it takes values in $V$. We assume
A0. $X, U$ are closed bounded convex sets, with nonempty interior

A1. the disturbance $v(t)$ is unknown but admissible for all $t$. 
A2. $0 \in V$ and $\exists x_{e} \in \operatorname{int}(X), u_{e} \in \operatorname{int}(U): x_{e}=$ $A x_{e}+B u_{e}$.

A3. the state $x(t)$ is observed at all $t$.

We recall

Definition 1. $X$ is control invariant for (1) if for any $x \in X$ there exists a feasible control such that $x^{+} \in X$ for all admissible disturbances.

We use repeatedly the notion of a reach set $\mathcal{R}(X)$. With reference to system $(1), \mathcal{R}(X)$ is the set of states that can reach $X$ in one step with a feasible control for all admissible disturbances.

As is well known - and easy to check - control invariance of $X$ holds if and only if

$$
X \subset \mathcal{R}(X) .
$$

To emphasize the role of disturbances, (2) can be written alternatively

$$
X \subset \mathcal{R}_{0}(X-C V)
$$

where $\mathcal{R}_{0}(X-C V)$ is the reach set of $X-C V$ under zero disturbance. A property of $\mathcal{R}$ (shared by $\mathcal{R}_{0}$ ) is monotonicity

$$
\mathcal{A} \subset \mathcal{B} \quad \Rightarrow \quad \mathcal{R}(\mathcal{A}) \subset \mathcal{R}(\mathcal{B})
$$

meaning that a feasible control able to reach $\mathcal{A}$ also reaches $\mathcal{B}$.

Remark 1. If the origin is contained in $U$ and in the interior of $X$ and $C V$ inclusion (2) in absence of noise implies contractivity as well as control invariance of $X$, e.g. there exists $\lambda \in\left(\begin{array}{ll}0 & 1\end{array}\right)$ such that under a feasible control $X$ maps into $\lambda X$ at each step. This ensures asymptotic stabilizability to the origin for (1).

Definition 2. $X_{0} \subset X$ is safe wrt $X$ for (1) if for any initial state $x \in X_{0}$ there exist feasible controls such that all subsequent states are in $X$ for all admissible disturbances.

We state without proof the properties

Proposition 1. Union of control invariant (safe wrt $X$ ) sets is control invariant (safe wrt $X$ ). Intersection of control invariant sets need not be control invariant. Subsets of safe sets are safe, so their intersection is either empty or safe.

Hence a set containing a control invariant subset contains a maximal control invariant subset.

Proposition 2. The largest safe set $\hat{S}$ wrt $X$ is control invariant and it coincides with the maximal control invariant set contained in $X$.
Proposition 3. If $X\left(X_{0} \subset X\right)$ is invariant (safe) for (1) with controls in $U$ so is with controls in $\hat{U}$ for $\hat{U} \supset U$; if $X\left(X_{0} \subset X\right)$ is invariant (safe) with disturbance in $V$ so is with disturbance in $\hat{V}$ for $\hat{V} \subset V$. In the linear case, if $(X, U, V)$ is an invariant triple (meaning $X$ is invariant for (1) with controls in $U$ and disturbance in $V$ ) so is any triple $\left(\lambda\left(X-x_{e}\right), \mu\left(U-u_{e}\right), \nu V\right)$ for $0 \leq \nu \leq \lambda \leq \mu, x_{e} \in X, u_{e} \in U$ with $x_{e}=A x_{e}+$ $B u_{e}$.

\section{3. $K$-STEP INVARIANCE}

As discussed in the introduction invariance can be weakened by the notion of $k$-step invariance

Definition 3. Set $X$ is $k$-step control invariant for (1) if for any $x(0) \in X$ there exists a sequence of feasible controls $u^{k}=u(0) \ldots u(k-1)$ such that $x(k) \in X$ for all admissible disturbances $v^{k}=v(0) \ldots v(k-1)$.

Setting $\mathcal{S}^{1}(\cdot)=\mathcal{R}(\cdot)$ and $\mathcal{S}^{i+1}(\cdot)=\mathcal{R}\left(\mathcal{S}^{i}(\cdot)\right)$ we define

$$
\mathcal{R}^{k}(\cdot)=\cup_{i=1}^{k} \mathcal{S}^{i}(\cdot)
$$

and interpret $\mathcal{R}^{k}(X)$ as the set of states that under feasible controls reach $X$ in at most $k$ steps, for all admissible disturbances. Then $k$-step control invariance of $X$ holds if and only if

$$
X \subset \mathcal{R}^{k}(X) .
$$

The definition of $k$-step invariance does not rule out $\tau$-step invariance for $\tau<k$. In particular, 1-step invariance is the same as invariance.

To emphasize the role of disturbances, (4) can be written alternatively

$$
X \subset \mathcal{R}_{0}^{k}\left(X-\mathcal{D}^{k}\right)
$$

where $\mathcal{D}^{k}$ is the set of states reachable from 0 in $k$ steps by admissible disturbances under zero control and $\mathcal{R}_{0}^{k}(\cdot)$ is the set of states that reach $(\cdot)$ in at most $k$ steps with feasible controls under zero disturbances. Notice that $\mathcal{R}^{k}, \mathcal{R}_{0}^{k}$ inherit the monotonicity of $\mathcal{R}, \mathcal{R}_{0}$ meaning that a sequence of feasible controls able to reach $\mathcal{A}$ in at most $k$ steps also reaches $\mathcal{B} \supset \mathcal{A}$ in at most $k$ steps.

A control invariant set need not be $k$-step control invariant, since $u^{k}$ in Def. 3 is open loop. To illustrate, for

$$
x^{+}=\frac{3}{4} x+u+v, \quad|u| \leq \frac{1}{2}, \quad|v| \leq 1
$$

set $X=\{x:|x| \leq 2\}$ is control invariant under $u=-\min \left\{\frac{3}{4}|x|, \frac{1}{2}\right\} \operatorname{sign}(x)$, but not 2 -step control 
invariant as there are points of $X$ that cannot be brought to 0 with two feasible controls while two admissible disturbances can take the state outside $X$ from all points except 0 . However, in absence of disturbance $(v \equiv 0)$ a control invariant set is $k$-step control invariant for any $k>1$.

The example enlights a simple general property.

Proposition 4. If $\mathcal{D}^{k} \subset \mathcal{R}_{0}^{k}(0)$ then $\mathcal{R}_{0}^{k}(0)$ is $k$ step control invariant.

Proof Since $\{0\} \subset \mathcal{R}_{0}^{k}(0)-\mathcal{D}^{k}$, from the monotonicity of $\mathcal{R}_{0}^{k}$ we have

$$
\mathcal{R}_{0}^{k}(0)=\mathcal{R}_{0}^{k}(\{0\}) \subset \mathcal{R}_{0}^{k}\left(\mathcal{R}_{0}^{k}(0)-\mathcal{D}^{k}\right)
$$

and the conclusion follows from (5) with $X=$ $\mathcal{R}_{0}^{k}(0)$

In absence of disturbance a known result Aubin [1991], Caravani, De Santis [2002] is that a set $X$ contains a control invariant subset for (1) if and only if it contains a feasible equilibrium point of (1), a point $x_{e}$ satisfying $x_{e}=A x_{e}+B u_{e}$ for some $u_{e} \in U$. In presence of disturbance sufficiency fails and we have the following

Proposition 5. Set $X$ contains a control invariant subset for (1) if and only if it contains a set of the form $x+C V$ which is safe wrt $X$.

Proof (if) Assume $x+C V \subset X$ is safe wrt $X$. As points of the reach set of a safe set are safe, and union of safe sets is safe, a maximal safe set contained in $X$ exists. But the maximal safe set is control invariant.

(only if) Assume there exists no $x$ such that $x+$ $C V \subset X$. This implies

$$
\forall x \exists v \in V: x+C v \notin X .
$$

Then for any $x \in X$ and all $f: X \mapsto U$ there exists $v \in V$ such that $A x+B f(x)+C v \notin X$, i.e. $X$ cannot contain a control invariant subset. Thus we proved that a set of the form $x+C V$ must be contained in any $X$ containing a control invariant subset; in particular, it must be contained in a control invariant subset of $X$. This shows $x+C V$ must be safe wrt $X$.

Proposition 6. Set $X$ contains a $k$-step control invariant subset for (1) if and only if it contains a set of the form $x+\sum_{t=1}^{k} A^{k-t} C V$ (with the sum meant in a set-theoretic sense) which is safe wrt $X$.

Proof (same as Prop. 5, with appropriate replacement of the variables)
Notice that this also establishes

Proposition 7. A set $X$ containing a $k$-step control invariant subset for (1) also contains a control invariant subset.

Proof By Prop. 6, $X$ contains a set of the form $x+\sum_{t=1}^{k} A^{k-t} C V$ which is safe wrt $X$ for (1). As $x+\sum_{t=1}^{\bar{k}} A^{k-t} C V \supset x+C V$, the latter is safe wrt $X$ for (1) and by Prop. 5 it contains a control invariant subset for (1).

In general, the condition of Prop. 5 cannot be checked in a finite number of steps. However,

Proposition 8. Let $X_{0}$ be $k$-step control invariant for system (1) and safe wrt $X$. Then a control invariant set contained in $X$ can be found in $k$ steps.

Proof Consider the algorithm

$$
\begin{aligned}
& S_{k}=X_{0} \\
& S_{\tau}=\operatorname{conv}\left(\mathcal{R}\left(S_{\tau+1}\right) \cup S_{\tau+1}\right) \cap X, \tau=k-1 \ldots 1 .
\end{aligned}
$$

$S_{k}$ is $k$-step control invariant and safe wrt $X$. Assume $S_{\tau+1}$ is $\tau+1$ step control invariant and safe wrt $X$. Under suitable controls, trajectories starting in $S_{\tau+1}$ at step 1 return to it at step $r \leq \tau+1$, therefore at step $r-1$ they are either in $S_{\tau+1}$ or in $\mathcal{R}\left(S_{\tau+1}\right) \cap X$. Therefore $\left(\mathcal{R}\left(S_{\tau+1}\right) \cup\right.$ $\left.S_{\tau+1}\right) \cap X$ is $\tau$-step control invariant. It is also safe wrt $X$, because $S_{\tau+1}$ is safe wrt $X$ and from $\mathcal{R}\left(S_{\tau+1}\right) \cap X$ it is possible to go to $S_{\tau+1}$ in one step. Due to linearity, if a set is invariant and safe so is its convex extension, hence $S_{\tau}$ is $\tau$-step control invariant and safe wrt $X$. By induction, $S_{1}$ is control invariant and safe wrt $X$, hence contained in $X$.

Proposition 9. Assume $(A, B)$ reachable in (1) and let $0 \in \operatorname{int}(U)$ and $0 \in \operatorname{int}(X)$. Then there exist a set $X_{0}$ and scalars $\lambda_{0}, \lambda_{s}$ such that $\lambda_{0} X_{0}$ is $n$-step invariant for (1) with controls in $\lambda_{0} U$ and disturbance in $V$ and safe wrt $\lambda_{s} X$.

Proof Let $P_{n}$ be the reachability matrix of $(A, B)$. The set of states of (1) that reach the origin in $n$ steps with feasible controls and no disturbance is

$$
\mathcal{R}_{0}^{n}(0)=\left\{x: \exists u^{n} \in U^{n}: A^{n} x+P_{n} u^{n}=0\right\} .
$$

We show that $X_{0}=\mathcal{R}_{0}^{n}(0)$ has the desired property. Since $P_{n}$ has rank $n$ there exists a matrix $Q$ such that $u^{n}=-Q A^{n} x, P_{n} Q=I$. Therefore $X_{Q}=\left\{x:-Q A^{n} x \in U^{n}\right\} \subset X_{0}$ and since $0 \in \operatorname{int}\left(U^{n}\right), 0 \in \operatorname{int}\left(X_{Q}\right) \subset \operatorname{int}\left(X_{0}\right)$. Hence $\exists \nu^{*}>0$ such that 
$\nu\left(C V+A C V+\cdots+A^{n-1} C V\right) \subset X_{0} \quad \forall \nu \in\left[0 \nu^{*}\right]$

that is, $\nu^{*} \mathcal{D}^{n} \subset \mathcal{R}_{0}^{n}(0)$. By Prop. $4, X_{0}$ is $n$-step invariant for (1) with controls in $U$ and disturbance in $\nu^{*} V$. By Prop. $3, \lambda_{0} X_{0}$ is $n$-step invariant with controls in $\lambda_{0} U$ and disturbance in $V$ for $\lambda_{0}=1 / \nu^{*}$. Thus under arbitrary disturbances in $V$ there exist controls in $\lambda_{0} U$ yielding $x(0), x(\tau) \in$ $\lambda_{0} X_{0}$ for some $\tau \leq n$ in (1). Let $T$ be the set of all such trajectories. Clearly $0 \in \lambda_{0} X_{0} \subset T$ and since trajectories of (1) have finite amplitude in finite time, $T$ is bounded. As $X$ has a nonempty interior containing the origin, there exists a $\lambda_{s}<\infty$ such that $T \subset \lambda_{s} X$, e.g. $\lambda_{0} X_{0}$ is safe wrt $\lambda_{s} X$.

Remark 2. The set $X_{0}$ used in the proof is actually the largest set of states that reaches the origin in $n$ steps with feasible controls. In practice, any set sharing this property - for example $X_{Q}{ }^{-}$ serves the same purpose.

Proposition 10. Let $\hat{S}$ be the maximal invariant subset of $X$ with controls in $U$ and disturbance in $V$ and let $x_{e} \in \hat{S}$ where $x_{e}=A x_{e}+B u_{e}$ be a feasible equilibrium point of (1). Let $\lambda^{*}$ be the infimum $\lambda$ such that $\lambda\left(X-x_{e}\right)$ contains an invariant subset with controls in $\lambda\left(U-u_{e}\right)$ and disturbance in $V$. Then $X^{*}=\lambda^{*}\left(X-x_{e}\right)$ contains a control invariant subset if and only if it contains $S^{*}=\lambda^{*}\left(\hat{S}-x_{e}\right)$, the maximal control invariant subset of $X^{*}$.

Proof By assumption, $x_{e}=A x_{e}+B u_{e}$ for some $u_{e} \in U$. By Prop. 3, if $(S, U, V)$ is an invariant triple for (1), there are values of $\lambda$ such that $\left(S-x_{e}, U-u_{e}, V / \lambda\right)$ keeps the property. Let $\lambda^{*}$ be the infimum of such values. Clearly $\lambda^{*} \leq 1$. Consider $X^{*}=\lambda^{*}\left(X-x_{e}\right)$. This set contains an invariant subset with controls in $U^{*}=$ $\lambda^{*}\left(U-u_{e}\right)$ and disturbance in $V$ if and only if it contains a maximal invariant subset under the same constraints. Assume the latter is $S^{\prime} \neq S^{*}=$ $\lambda^{*}\left(\hat{S}-x_{e}\right)$. If $S^{\prime}$ is an invariant subset of $X^{*}$ with controls in $U^{*}$ and disturbance in $V$, by Prop. $3 S^{\prime} / \lambda^{*}+x_{e}$ is an invariant subset of $X$ with controls in $U$ and disturbance in $V$. But $S^{\prime} / \lambda^{*}+$ $x_{e} \neq S^{*} / \lambda^{*}+x_{e}=\hat{S}$ and $\operatorname{conv}\left(\hat{S} \cup S^{\prime} / \lambda^{*}+x_{e}\right)$ is a control invariant subset of $X$ properly including $\hat{S}$ against the assumption.

\section{DOES $X$ CONTAIN A CONTROL INVARIANT SUBSET?}

The question whether a set $X$ contains or not a control invariant subset can be answered by the internal invariant approximation algorithm as follows. Assume $0 \in \operatorname{int}(X)$ is the feasible equilibrium point of interest.

\section{Algorithm 1}

1. Find a set $X_{0}$ and a scalar $\lambda_{0}$ such that $\lambda_{0} X_{0}$ is $n$-step invariant with controls in $\lambda_{0} U$ and disturbance in $V$.

2. Find a scalar $\lambda_{s} \geq \lambda_{0}$ such that $\lambda_{0} X_{0}$ is safe wrt $\lambda_{s} X$

3. Using Prop. 8 find $S$, an invariant subset of $\lambda_{s} X$ with controls in $\lambda_{s} U$ and disturbance in $V$.

4. Using the internal approximation algorithm, find $\hat{S}$, the maximal invariant subset of $\lambda_{s} X$ with controls in $\lambda_{s} U$ and disturbance in $V$.

5. Using Prop. 11 find $\lambda^{*}$. If $\lambda^{*} \lambda_{s} \leq 1$ conclude that $\lambda^{*} \hat{S}$ is the largest control invariant subset of $X$; otherwise no invariant set with controls in $U$ and disturbance in $V$ is contained in $X$.

A few remarks are in order. Steps 1 and 2 are based on Prop. 9, whose proof also suggests a way to compute $\lambda_{0}, \lambda_{s}, X_{0}$. Step 4 may require infinite iterations of the internal invariant approximation algorithm. However, truncation at iteration $k<$ $\infty$ yields useful information since $\lambda_{k} \lambda_{s} X$ contains a control invariant subset. More specifically, replace 4 and 5 by

4k. Find $\lambda^{*}=\inf \lambda: \lambda S$ is invariant with controls in $\lambda \lambda_{s} U$ and disturbance in $V$.

5k. While $\lambda^{*} \lambda_{s}>1$ repeat:

$$
\begin{aligned}
& S \Leftarrow \mathcal{R}\left(\lambda^{*} S\right) \cap \lambda^{*} \lambda_{s} X \\
& \lambda^{*} \Leftarrow \inf \lambda: \lambda S \text { is invariant with controls }
\end{aligned}
$$
in $\lambda \lambda_{s} U$ and disturbance

in $V$.

The $S$-assignment in $5 \mathrm{k}$ corresponds to one step of the internal invariant approximation algorithm and it yields a larger invariant set than $\lambda^{*} S$ contained in $\lambda^{*} \lambda_{s} X$. Notice that since $S$ at step 3 is an invariant subset of $\lambda_{s} X, \lambda^{*}$ is always $\leq 1$ in $4 \mathrm{k}$. Thus the sequence of sets $\left\{\lambda^{*} \lambda_{s} X\right\}$ generated in $5 \mathrm{k}$ is decreasing and it converges to the smallest set in this family containing an invariant subset with controls in $U$ and disturbances in $V$. The algorithm stops if $\lambda^{*} \lambda_{s} \leq 1$ in which case $X$ contains an invariant subset $\lambda^{*} S$ with controls in $U$ and disturbances in $V$.

We remark that the procedure outlined, contrary to De Santis et al. [2004], does not require $C V$ to be a $\mathcal{C}$-set (a convex compact set with a nonempty interior containing the origin) and it appears simpler to implement. When $X, U, V$, are specified as convex polyhedra, Algorithm 1 reduces to a sequence of linear programs and elementary operations on convex sets. These have been performed using the Geometric Bounding Toolbox developed by Veres [2002]. The detailed procedure will be included in an extended version of the paper. 


\section{AN EXAMPLE}

Consider the double integrator used in Raković et al. [2005].

$$
x^{+}=\left[\begin{array}{ll}
1 & 1 \\
0 & 1
\end{array}\right] x+\left[\begin{array}{l}
1 \\
1
\end{array}\right] u+v
$$

with

$$
\begin{gathered}
X=\left\{x:-3 \leq x_{1} \leq 1.85 ;-3 \leq x_{2} \leq 3\right. \\
\left.x_{1}+x_{2} \geq-2.2\right\} \\
U=\{u:|u| \leq 2.4\} \quad V=\left\{v:|v|_{\infty} \leq 1\right\} .
\end{gathered}
$$

We have $X=a b c d e$ in Fig.1. Step 1 of the algorithm finds $\lambda_{0} X_{0}=A B C D\left(\lambda_{0}=2.0833\right)$ to be 2-step invariant with controls in $\lambda_{0} U$ and disturbance in $V$. At Step $2 A B C D$ is found safe wrt $\lambda_{s} X=L E F G H\left(\lambda_{s}=3.333\right)$. At Step 3 we find an invariant $S=A M N P C R H L$ contained in $\lambda_{s} X$. At Step $4 \mathrm{k}$ we find a contraction of this set with $\lambda^{*}=0.3$ which (since $\lambda^{*} \lambda_{s}<1$ ) is contained in $X$ with controls in $U$ and disturbance in $V$, see convex polygon inside abcde in Fig. (a): we conclude $X$ contains a control invariant subset. An improved estimate to the maximal control invariant subset of $X$ could obtained by performing Step 5k of the algorithm. However, the maximal invariant set inside abcde in Fig (b) (easy to compute in this particular case) shows that the first iteration of the algorithm already provides a good appromation (inner polygon in Fig. (b)).

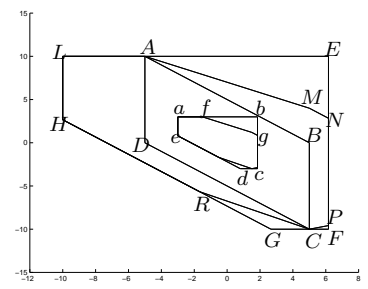

(a) Invariant Set Computation

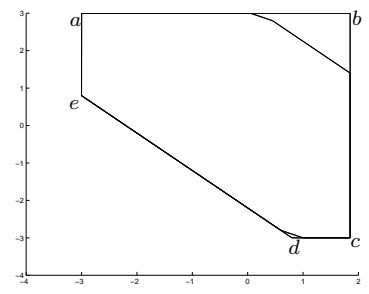

(b) Maximal Invariant Set

\section{CONCLUSION}

In this paper we have presented a technique to evaluate the maximal control invariant subset of a given set for LDT systems. The approach improves the state of current research in the following aspects: 1 . it quickly provides a first approximate solution consisting of an "inflated" version of the given set containing a control invariant subset; in subsequent iterations, the size of the inflated set is reduced and the procedure is shown to converge to the smallest set in the family containing a control invariant subset. This way, the question whether a set contains a control invariant subset is also answered algorithmically. 2. The assumptions necessary to obtain the desired result are minimal - in particular, it is not requested that the disturbance vector acts on every state component.

\section{REFERENCES}

Aubin J.P. (1991) Viability Theory. Boston: Birkhäuser.

Blanchini F., Miani S. (1999b) A universal class of smooth functions for robust control, IEEEAC-44-3, pp. 641-47.

Blanchini F. (1999a) Set Invariance in Control, Automatica 35 pp. 1747-67.

Caravani P., De Santis E. (2002) Doubly Invariant Equilibria of Linear Discrete-Time Games, Automatica 38-02 pp. 1531-38.

De Santis E. (1997) On Invariant Sets for Constrained Discrete Time Linear Systems with Disturbances and Parametric Uncertainties, Automatica 33-11, pp. 2033-39.

De Santis E., Di Benedetto M.D., Berardi L. (2004) Computation of Maximal Safe Sets for Switching Systems, IEEE-AC, 49-2, pp. 184195.

Farina L., Benvenuti L. (1998) Invariant Polytopes of Linear Systems, IMA J. Math. Control Info., vol. 15, pp. 233-40.

Gutman P. O., Cwikel M. (1987) An algorithm to find maximal state constraint sets for discretetime linear dynamical systems with bounded controls and states, IEEE-AC, 32-3, pp. 25154 .

Kerrigan E. C. (2000) Robust Constraint Satisfaction: Invariant Sets and Predictive Control, Ph.D. Thesis, University of Cambridge. Downloadable from http://wwwcontrol.eng.cam.ac.uk/eck21

Lasserre J. B. (1993) Reachable, Controllable Sets and Stabilizing Control of Constrained Linear Systems, Automatica 29-02, pp.5312-36.

Mayne D.Q. (2001) Control of constrained dynamic systems, Europ. J. of Ctrl. 7,pp. 87-99

Raković S. V., Mayne D. Q. , Kerrigan E. C. and Kouramas K. I. (2005) Optimized robust control invariant sets for constrained linear discrete - time systems, Proceedings of the 16th IFAC World Congress IFAC 2005,pp. na

Shamma J.S. (1996) Optimization of the $l^{\infty}$ Induced Norm Under Full State Feedback, IEEE-AC 41-4 pp. 533-44.

Veres S. M. (2002) Error Control in Polytope Computations, JOTA 113-2, pp. 325-55. 